\title{
Exogenous spermine attenuates diabetic kidney injury in rats by inhibiting AMPK/mTOR signaling pathway
}

\author{
XINYING ZHANG $^{1 *}$, LI ZHANG $^{2 *}$, ZHE CHEN $^{3}$, SIWEI LI $^{1}$, BINGBING CHE ${ }^{1}$, \\ NINGNING WANG ${ }^{1}$, JUNTING CHEN ${ }^{4}, \mathrm{CHANGQING} \mathrm{XU}^{1}$ and CAN WEI ${ }^{1}$ \\ ${ }^{1}$ Department of Pathophysiology, Harbin Medical University, Harbin, Heilongjiang 150086; \\ ${ }^{2}$ Department of Rehabilitation, The Second Affiliated Hospital of Heilongjiang University of Chinese Medicine, \\ Harbin, Heilongjiang 150001; ${ }^{3}$ Department of Infectious Diseases, General Hospital for The Head Office of \\ Agricultural Cultivation of Heilongjiang, Harbin, Heilongjiang 150088; ${ }^{4}$ Department of Anesthesiology, \\ The Fourth Affiliated Hospital of Harbin Medical University, Harbin, Heilongjiang 150081, P.R. China
}

Received July 29, 2020; Accepted January 19, 2021

DOI: $10.3892 / \mathrm{ijmm} .2021 .4860$

\begin{abstract}
Diabetic nephropathy (DN) is the primary cause of end-stagerenaldisease, which isclosely associated with dysfunction of the podocytes, the main component of the glomerular filtration membrane; however, the exact underlying mechanism is unknown. Polyamines, including spermine, spermidine and putrescine, have antioxidant and anti-aging properties that are involved in the progression of numerous diseases, but their role in DN has not yet been reported. The present study aimed to explore the role of polyamines in $\mathrm{DN}$, particularly in podocyte injury, and to reveal the molecular mechanism underlying the protective effect of exogenous spermine. Streptozotocin intraperitoneal injection-induced type 1 diabetic (T1D) rat models and high glucose (HG)-stimulated podocyte injury models were established. It was found that in T1D rat kidneys and HG-induced podocytes, ornithine decarboxylase (a key enzyme for polyamine synthesis) was downregulated, while spermidine/spermine N1-acetyltransferase (a key enzyme for polyamines degradation) was upregulated, which suggested that reduction of the polyamine metabolic pool particularly decreased spermine content, is a major factor in DN progression. In addition, hyperglycemia can induce an increased rat kidney weight ratio, serum creatinine, urea, urinary albumin excretion and glomerular cell matrix levels, and promote mesangial thickening and loss or fusion of podocytes. The
\end{abstract}

Correspondence to: Professor Changqing Xu or Dr Can Wei, Department of Pathophysiology, Harbin Medical University, 157 Baojian Road, Harbin, Heilongjiang 150086, P.R. China

E-mail: xucq45@126.com

E-mail: canwei528@163.com

*Contributed equally

Key words: diabetic nephropathy, podocyte, spermine, autophagy, AMPK/mTOR pathway expression levels of podocyte marker proteins (nephrin, CD2-associated protein and podocin) and autophagy-related proteins [autophagy protein 5 , microtube-associated proteins 1A/1B light chain 3 (LC3)II/LC3I, Beclin 1 and phosphorylated (p)-AMPK] were downregulated, while cleaved caspase-3, P62 and p-mTOR were increased. These changes could be improved by pretreatment with exogenous spermine or rapamycin (autophagic agonist). In conclusion, spermine may have the potential to prevent diabetic kidney injury in rats by promoting autophagy via regulating the AMPK/mTOR signaling pathway.

\section{Introduction}

Diabetic nephropathy (DN) is a major microvascular complication of diabetes that can lead to end-stage renal disease $(1,2)$. The pathogenesis of DN is remarkably complex, involving hyperglycemia-mediated intracellular metabolism disorders, autophagy, oxidative stress and endoplasmic reticulum stress, thereby resulting in increased mesangial matrix, thickened basement membrane and extensive, persistent proteinuria $(3,4)$. However, the underlying mechanisms have not yet been elucidated to date, and there are currently no effective prevention or treatment strategies. Podocytes are highly differentiated epithelial cells that enclose glomerular capillaries and are involved in maintaining the glomerular filtration barrier $(5,6)$. The structural integrity of podocytes is crucial for preventing the leakage of albumin and microalbuminuria $(7,8)$. At present, the mechanism of podocyte dysfunction in chronic kidney disease has been attracting increasing attention.

Polyamines (spermine, spermidine and putrescine) in humans, which are absorbed by the small intestine, are mainly produced by food intake, cell synthesis and intestinal microorganisms $(5,6)$. Polyamines have a variety of biological functions, including regulating cell proliferation, differentiation and apoptosis $(7,8)$. Polyamine metabolism, the abnormalities of which affect the progression of cardiovascular and kidney diseases, is mainly regulated by ornithine decarboxylase (ODC) and spermidine/spermine 
N1-acetyltransferase (SSAT) (9). Among them, spermine has the strongest biological activity, which can play a protective role in the progression of diabetic cardiomyopathy, myocardial hypertrophy and renal ischemia/reperfusion (I/R) injury by promoting autophagy, inhibiting inflammatory reactions, oxidative stress and endoplasmic reticulum stress, and regulating calcium homeostasis (10-12). However, whether DN and podocyte damage are implicated in abnormal polyamine metabolism in the kidney is unclear, and no relevant studies have been conducted thus far.

In the present study, type 1 diabetic (T1D) rats and high glucose (HG)-induced podocyte injury were investigated; the association between polyamine homeostasis and autophagy and the related signaling pathways were examined. In addition, exogenous polyamine treatment was used to observe the central role of abnormal polyamine metabolism in the pathogenesis of DN. These results may help elucidate the pathogenesis of DN from a novel perspective and suggest future strategies for the prevention and treatment of this condition.

\section{Materials and methods}

Animal and model. Male Wistar rats (age, 8 weeks old; weight, 200-250 g) were purchased from the animal care facility at the Second Affiliated Hospital of Harbin Medical University (Harbin, China). All animal experimental protocols complied with the Guide for the Care and Use of Laboratory Animals published by the National Institutes of Health (13). The present study was approved by the Institutional Animal Research Committee of Harbin Medical University. All animals were housed at the animal care facility of Harbin Medical University at $25^{\circ} \mathrm{C}$ with a $12 / 12 \mathrm{~h}$ light/dark cycle in a vivarium with humidified airflow, and were allowed free access to normal rat chow and water throughout the study period.

Diabetes can be induced by an intraperitoneal of streptozotocin (STZ). A total of 45 rats were randomly divided into three groups: i) Control ( $n=15), 0.1 \mathrm{M}$ sterile citrate buffer ( $\mathrm{pH} 4.5)$ was injected intraperitoneally as a control at the same volume as that in the model group; ii) T1D $(n=15)$, a single intraperitoneal injection of STZ $(60 \mathrm{mg} / \mathrm{kg}$, dissolved in $0.1 \mathrm{M}$, $\mathrm{pH} 4.5$ citric acid-citrate sodium buffer) was used to establish the T1D model. When the concentration of serum glucose was $>16.7 \mathrm{mmol} / \mathrm{l}$, the T1D model was considered to be established successfully (14); and iii) T1D + Sp ( $=15)$, spermine solution $(2.5 \mathrm{mg} / \mathrm{kg} / \mathrm{day}$, dissolved in normal saline) was injected intraperitoneally every day for 2 weeks before STZ injection, followed by an injection of spermine $(2.5 \mathrm{mg} / \mathrm{kg} /$ day $)$ every other day for 12 weeks. Rats in each group were euthanized at week 12 of modeling. Under anesthesia with intraperitoneal $2 \%$ pentobarbital $(50 \mathrm{mg} / \mathrm{kg}$, dissolved in normal sodium), the kidneys were removed by opening the abdomen and $0.1 \%$ lidocaine ( $2 \mathrm{mg} / \mathrm{kg}$, dissolved in normal saline) was used to infiltrate the incision wound for analgesia. The rats were sacrificed after surgery with an overdose of $2 \%$ pentobarbital (100 mg/kg injected intravenously) (15).

Measurement of biochemical parameters. Blood glucose and body weight were measured at weeks $0,4,8$ and 12 in each group. Urine samples were collected for $24 \mathrm{~h}$ on week 12 , while blood was collected from the retro-orbital vein by removing the eyeballs. Subsequently, the rats were euthanized, and the kidneys were then removed and weighed. The levels of urinary albumin excretion (UAE; cat. no. ZK-H1678), serum creatine (Scr; cat. no. GMS70021.7) and urea (cat. no. GMS70022.1) were measured using appropriate ELISA kits (Shenzhen Ziker Biological Technology Co., Ltd.).

Kidney histology and immunohistochemistry. Sections of kidney tissues were fixed with $4 \%$ paraformaldehyde at room temperature for $48 \mathrm{~h}$, embedded in paraffin and sectioned into $4-\mu \mathrm{m}$ sections. Renal sections were stained at room temperature with hematoxylin and eosin (H\&E) for $3 \mathrm{~min}$, and with periodic acid-Schiff staining (PAS) for $30 \mathrm{sec}$ for $5 \mathrm{~min}$, and then sections were assessed by light microscopy. Images of 20 glomeruli per rat were obtained, and the PAS-positive (purple) area per glomeruli was quantified using ImageJ v1.8.0 software (National Institutes of Health).

For immunohistochemistry, paraffin-embedded sections were blocked with 5\% BSA (cat. no. SW3015; Beijing Solarbio Science \& Technology Co., Ltd.) for $30 \mathrm{~min}$ at room temperature and stained with an antibody against podocin (1:50; cat. no. ab50339; Abcam) at $4^{\circ} \mathrm{C}$ overnight, and then washed with PBS. Next, the mixture was incubated with the secondary antibody (goat anti-rabbit IgG; 1:500; cat. no. ab6721; Abcam) for $1 \mathrm{~h}$ at room temperature and washed with PBS. Finally, the sections were stained with a solution of diaminobenzidine for $2 \mathrm{~min}$ at room temperature, washed with PBS, dehydrated and permeabilized with xylene. Stained images (10 glomeruli per kidney) were visualized by light microscopy and all the images were analyzed with ImageJ software.

Cell culture and treatment. Rat podocytes (cat. no. BNCC338697; BeiNa Culture Collection; Beijing Beina Chunglian Biotechnology Research Institute) were cultured at $37^{\circ} \mathrm{C}$ in a $5 \% \mathrm{CO}_{2}$ humidified incubator in DMEM (Gibco; Thermo Fisher Scientific, Inc.) containing 10\% fetal bovine serum (FBS; Gibco; Thermo Fisher Scientific, Inc.) and 1\% penicillin. Podocytes were cultured for 6-12 h in DMEM containing 5.5 mM D-glucose (Sigma-Aldrich; Merck KGaA) without FBS before exposure to various experimental conditions. The cells were randomly divided into six groups: i) Normal-glucose group (NG), where the podocytes were incubated in DMEM containing $5.5 \mathrm{mM}$ glucose for $48 \mathrm{~h}$; ii) mannitol group $(\mathrm{M})$, where the podocytes were incubated in DMEM containing $40 \mathrm{mM}$ mannitol and $5.5 \mathrm{mM}$ glucose for $48 \mathrm{~h}$; iii) high-glucose group (HG), where the podocytes were incubated in DMEM containing $40 \mathrm{mM}$ glucose for $48 \mathrm{~h}$; iv) spermine-treated group $(\mathrm{HG}+\mathrm{Sp})$, where the podocytes were pretreated with $1,5,10,20$ and $50 \mu \mathrm{M}$ spermine (Sigma-Aldrich; Merck KGaA) for $2 \mathrm{~h}$ before being subjected to HG conditions; v) rapamycin-treated group (HG + Rap), where the podocytes were pretreated with $10 \mathrm{mM}$ rapamycin (MCE) for $2 \mathrm{~h}$ before being subjected to $\mathrm{HG}$ conditions; and vi) Compound $\mathrm{C}$ treatment group $(\mathrm{HG}+\mathrm{Sp}+$ Compound $\mathrm{C})$, where $10 \mathrm{mM}$ Compound $\mathrm{C}$ (Selleck Chemicals), an AMPK inhibitor, was added to the medium $1 \mathrm{~h}$ before $\mathrm{HG}$ and spermine addition.

Cell viability assay. Quantitative evaluation of cell viability was performed using a Cell Counting Kit-8 (CCK-8; Dojindo 
Molecular Technologies, Inc.) assay according to the manufacturer's instructions. Cells were seeded in 96-well plates at a concentration of $1 \times 10^{3}$ cells/well. After $24 \mathrm{~h}$ of treatment in each group of cells, $10 \mu \mathrm{l}$ CCK-8 was added to each well and incubated for $2 \mathrm{~h}$ at $37^{\circ} \mathrm{C}$. Next, the absorbance at $570 \mathrm{~nm}$ was determined using a microplate spectrophotometer reader.

Apoptosis assay by Hoechst 33342 staining. Hoechst 33342 is a blue, fluorescent dye that can penetrate the cell membrane and is used for apoptosis detection. Podocytes were plated into 24 -well dishes $\left(2 \times 10^{5}\right.$ cells/well $)$ and were cultured under the aforementioned conditions. Then, cells were rinsed with PBS and incubated for $10 \mathrm{~min}$ at room temperature using $5 \mathrm{mg} / \mathrm{ml}$ Hoechst 33342. Staining was observed by fluorescence microscopy (Nikon Corporation) at x400 magnification. The excitation wavelength was maintained at $380 \mathrm{~nm}$. When apoptosis occurred, the nuclei appeared as dense or fragmented.

Electron microscopy. The ultrastructure of the kidney was detected by electron microscopy. Samples of renal sections were cut into pieces $(<1 \mathrm{~mm})$ and fixed in $2.5 \%$ glutaraldehyde for $4 \mathrm{~h}$ at room temperature. Tissues were post-fixed in $1 \%$ osmium tetroxide with $0.1 \mathrm{M}$ sodium cacodylate, dehydrated through graded concentrations of ethanol and propylene oxide, and subsequently embedded in Epon 812 for $24 \mathrm{~h}$ at $60^{\circ} \mathrm{C}$. Ultrathin sections were cut from blocks and mounted on copper grids for counterstaining with lead citrate and uranium acetate for $7 \mathrm{~min}$ each at $75^{\circ} \mathrm{C}$. The sections were observed under a Philips CM 120 electron microscope (Philips Medical Systems, Inc.).

Immunoblotting. Rat renal tissues and rat podocytes were homogenized in $0.5 \mathrm{ml}$ RIPA buffer prior to being transferred to small tubes and rotated $20 \mathrm{sec}$ per $5 \mathrm{~min}$ six times. Solubilized proteins were collected after centrifugation at $10,000 \times \mathrm{g}$ at $4^{\circ} \mathrm{C}$ for $30 \mathrm{~min}$. The supernatant was collected and stored at $-80^{\circ} \mathrm{C}$. The protein concentration of each sample was quantified using an enhanced BCA protein assay kit. For detection of protein levels, protein lysates ( $40 \mu \mathrm{g}$ protein loaded per lane) from each group of cells and tissues were separated via SDS-PAGE on a $10 \%$ gel, and subsequently separated proteins were electrotransferred onto a PVDF membrane (EMD Millipore). After blocking with TBS with $0.1 \%$ Tween-20 (TBST) containing 5\% non-fat dry milk for $1 \mathrm{~h}$ at $4^{\circ} \mathrm{C}$, the membrane was incubated overnight at $4^{\circ} \mathrm{C}$ with antibodies against ODC (1:1,000; cat. no. ab97395; Abcam), SSAT (1:1,000; cat. no. ab105220; Abcam), nephrin (1:400; cat. no. BA1669; Boster Biological Technology), podocin (1:400; cat. no. BA3416; Boster Biological Technology), CD2-associated protein (CD-2AP; 1:1,000; cat. no. bs-0512R; BIOSS), microtube-associated proteins $1 \mathrm{~A} / 1 \mathrm{~B}$ light chain 3 (LC3; 1:1,000; cat. no. 3868T; Cell Signaling Technology, Inc.), Beclin 1 (1:1,000; cat. no. 3495T; Cell Signaling Technology, Inc.), P62 (1:1,000; cat. no. 8025T; Cell Signaling Technology, Inc.), autophagy protein 5 (Atg5; 1:1,000; cat. no. 12994T; Cell Signaling Technology, Inc.), cleaved caspase-3 (1:1,000; cat. no. 9661T; Cell Signaling Technology, Inc.), total (t)-mTOR (1:1,000; cat. no. A00003-2; Boster Biological Technology), phosphorylated (p)-mTOR (1:600; cat. no. BM4840; Boster Biological Technology), t-AMPK (1:1,000; cat. no. A00994-6;
Boster Biological Technology), and p-AMPK (1:1,000; cat. no. P00994; Boster Biological Technology). The membrane blots were washed and incubated at $4{ }^{\circ} \mathrm{C}$ with anti-rabbit (cat. no. ab6721) or anti-mouse (cat. no. ab6728) IgG antibodies (1:10,000; Abcam) for $1.5 \mathrm{~h}$. The signals were detected by an enhanced chemiluminescence kit (Thermo Fisher Scientific, Inc.) and the Multiplex Fluorescence Imaging System (ProteinSimple). The intensities of the protein bands were semi-quantified by AlphaView SA 3.0 software (ProteinSimple) and normalized to $\beta$-actin.

Immunofluorescence staining. Podocytes were plated into 24 -well dishes $\left(2 \times 10^{5}\right.$ cells/well $)$ and cultured under the indicated conditions. Cells were fixed with $4 \%$ paraformaldehyde at room temperature for $24 \mathrm{~h}$, permeabilized in $0.5 \%$ Triton X-100 for $30 \mathrm{~min}$ and blocked with $1 \%$ BSA for $1 \mathrm{~h}$ at $4^{\circ} \mathrm{C}$. Next, cells were washed and incubated with anti-nephrin antibodies (1:1,000; cat. no. ab216341; Abcam) overnight at $4^{\circ} \mathrm{C}$. Finally, the cells were incubated with a secondary antibody (goat anti-rabbit IgG; 1:1,000; cat. no. ab6721; Abcam) for $2 \mathrm{~h}$ at room temperature, and observed with a fluorescence microscope at x400 magnification (Nikon Corporation).

Statistical analysis. Data are expressed as the mean \pm standard error of the mean. Each measurement was obtained by performing $\geq 3$ independent experiments. Statistical comparisons were conducted using paired or unpaired t-tests or one-way ANOVA followed by Bonferroni post hoc test. $\mathrm{P}<0.05$ was considered to indicate a statistically significant difference.

\section{Results}

Spermine improves the general condition and renal function parameters of $T 1 D$ rats. The present study established a rat model of intraperitoneally injected STZ-induced diabetes. The results showed that, compared with those of the control group, the blood glucose and the kidney weight/body weight ratio of the T1D rats were increased (Fig. 1A and C), while their body weight was mildly decreased (Fig. 1B). The animals also developed common signs of T1D, including polydipsia, polyuria, and noticeable hypoactivity and weakness (data not shown). Renal function parameters were evaluated, and it was found that Scr, serum urea and UAE in the T1D group were significantly increased (Fig. 1D-F).

Next, the expression of ODC and SSAT was detected by immunoblotting. The results showed that the expression of ODC was decreased, while that of SSAT was increased, in the T1D group (Fig. 1G), indicating that the metabolism of polyamines was altered and the content of endogenous spermine was decreased. Therefore, exogenous spermine pretreatment was added in subsequent experiments. The results revealed that exogenous spermine could improve the general condition and renal function-related indicators in T1D rats, which suggested that spermine may exert a protective effect on diabetic renal function.

Spermine improves the morphological and ultrastructural changes of the kidney in T1D rats. PAS and H\&E staining were used to observe the morphological changes of the kidney. The results revealed moderate mesangial proliferation, matrix 
A

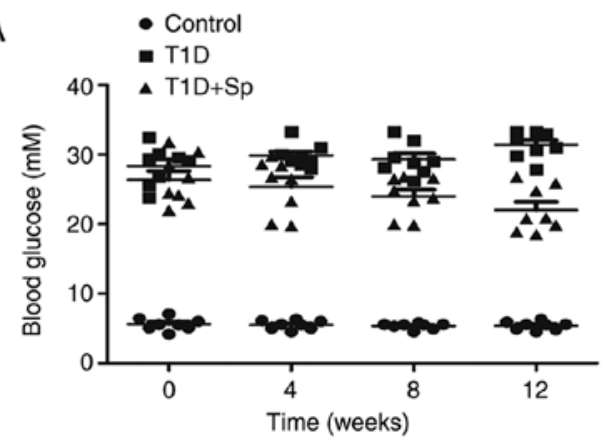

C

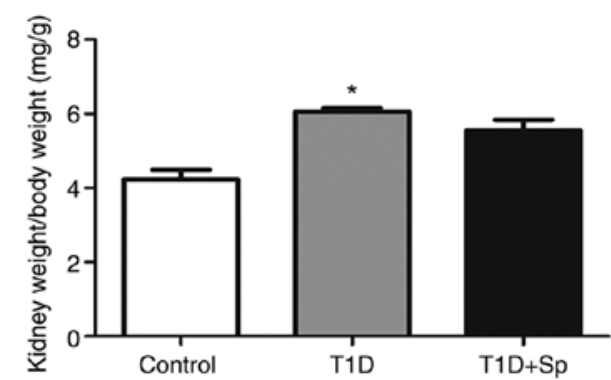

E

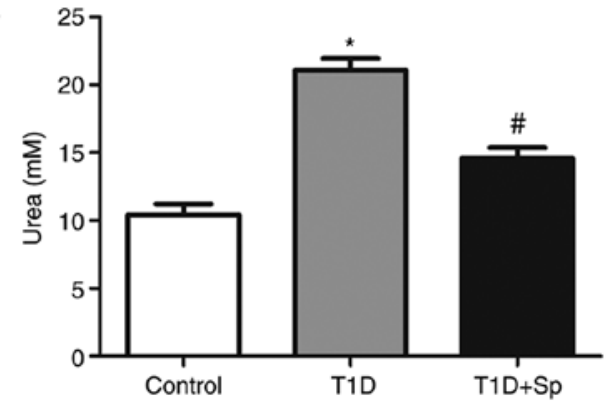

G

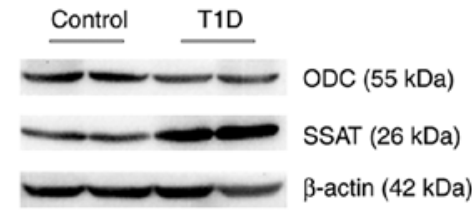

B

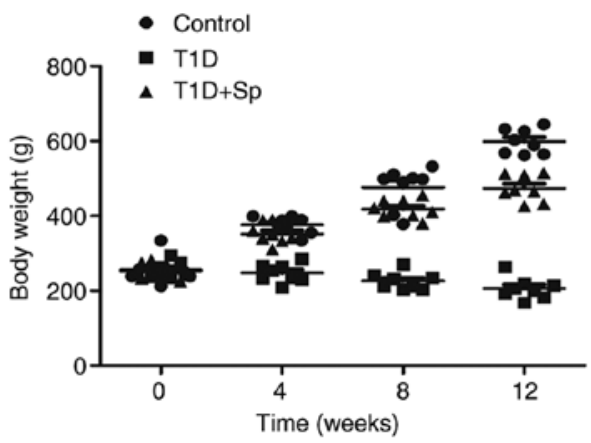

D

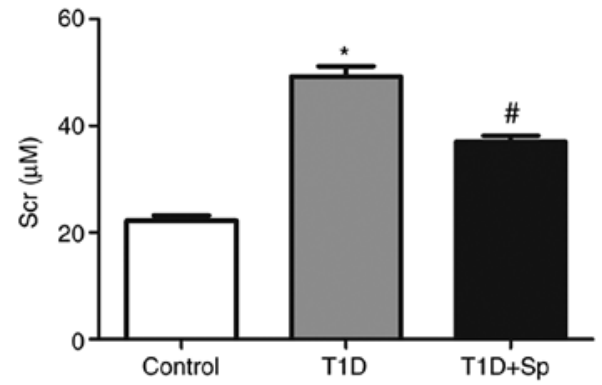

F
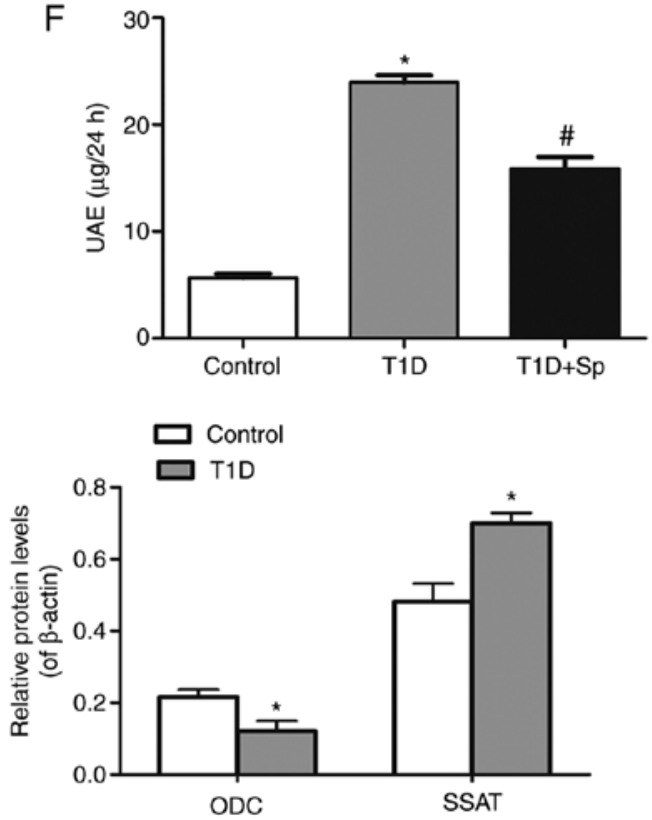

Figure 1. Spermine improves the general condition and renal function parameters of T1D rats. (A) Blood glucose levels and (B) body weight were measured at $0,4,8$ and 12 weeks after the STZ-induced diabetic rat model was established. (C) Kidney weight/body weight ratio, (D) Scr, (E) urea and (F) UAE were measured at 12 weeks in STZ-induced diabetic rats and spermine-pretreated rats. (G) Representative immunoblotting analysis of the protein levels of ODC and SSAT in STZ-induced rats. Data are expressed as the mean \pm standard error of the mean $(n=6) .{ }^{*} \mathrm{P}<0.05$ vs. the control group; ${ }^{*} \mathrm{P}<0.05$ vs. the T1D group. T1D, type 1 diabetic; STZ, streptozotocin; Scr, serum creatinine; UAE, urinary albumin excretion; ODC, ornithine decarboxylase; SSAT, spermidine/spermine N1-acetyltransferase; Sp, spermine-treated group.

accumulation and parietal endothelial cell proliferation in the T1D group, which further verified renal injury. These pathological changes were attenuated in the presence of spermine (Fig. 2A, B and D). Electron microscopic examination revealed that podocytes were disorganized with obvious loss and effacement of foot processes (FPs) and thickening of the glomerular basement membrane (GBM) in the T1D group, but these abnormalities were alleviated in the T1D + Sp group (Fig. 2C and E). Taken together, these results suggested that spermine may protect against diabetic kidney damage.
Spermine attenuates podocyte injury and promotes autophagy in TID rats. In vivo, the expression levels of podocyte-specific proteins, including nephrin, CD-2AP and podocin, were detected by immunoblotting and immunohistochemistry. The expression levels of podocyte-related proteins were significantly reduced in the T1D group (Fig. 3A). Immunohistochemical staining of podocin further supported the aforementioned results (Fig. 3B).

Next, the expression of autophagy-related proteins were examined. In comparison with the control group, the 
A

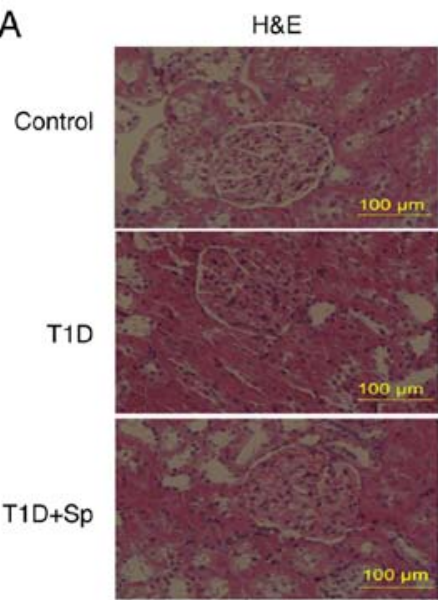

D

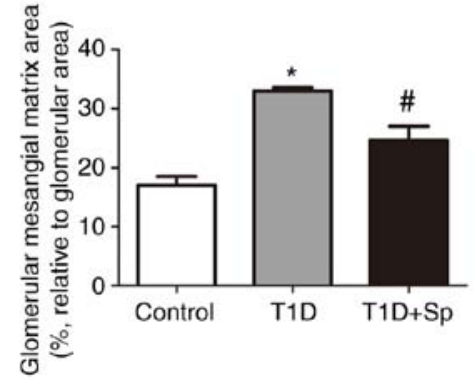

B
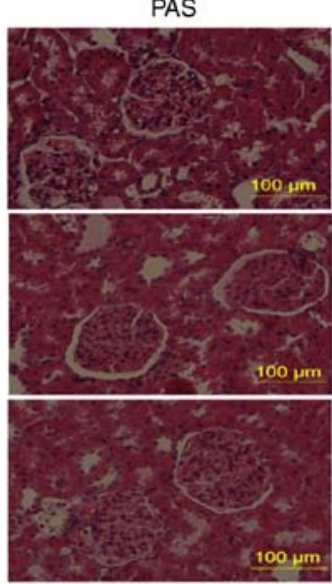

$\mathrm{E}$

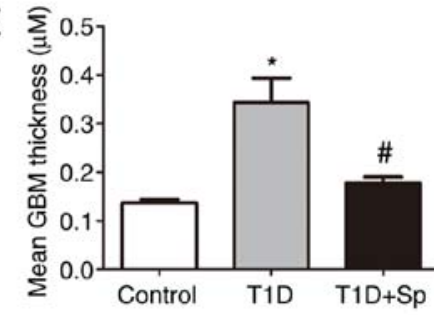

C
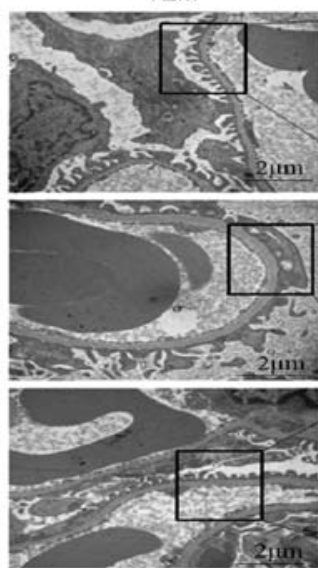
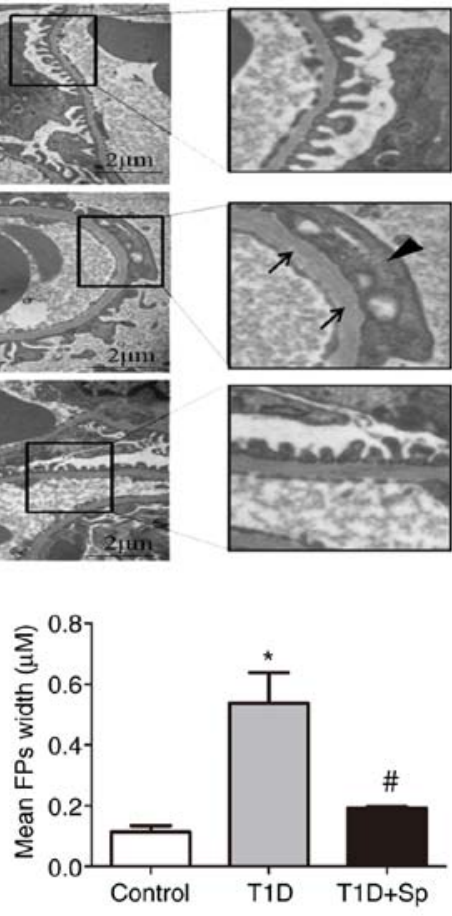

Figure 2. Spermine improves the morphological and ultrastructural changes of the kidney in T1D rats. (A) Representative images of H\&E staining (scale bar, $100 \mu \mathrm{m}$ ). (B) Representative images of PAS staining (Scale bar, $100 \mu \mathrm{m}$ ). (C) Representative TEM images (scale bar, $2 \mu \mathrm{m}$ ). (D) Quantification of the glomerular mesangial matrix area (percentage relative to the glomerular area) of the kidney sections. (E) Quantitative assessment of the GBM thickness (arrows) and FPs width (triangle). Data are expressed as the mean \pm standard error of the mean $(\mathrm{n}=6)$. ${ }^{*} \mathrm{P}<0.05$ vs. the control group; ${ }^{\sharp} \mathrm{P}<0.05$ vs. the T1D group. T1D, type 1 diabetic; H\&E, hematoxylin and eosin; PAS, periodic acid-Schiff; GBM, glomerular basement membrane; FPs, foot processes; TEM, transmission electron microscopy; Sp, spermine-treated group.

expression levels of Atg5, Beclin 1 and LC3II/LC3I were decreased and P62 was increased in the T1D group (Fig. 3C). Immunohistochemical staining revealed a mildly decreased expression of LC3 localized to glomeruli in T1D rats (Fig. 3D), which confirmed autophagy insufficiency in DN rats. These changes were obviously improved by exogenous spermine pretreatment. Collectively, these results indicated that the effect of spermine in DN was associated with activation of autophagy.

Spermine mitigates HG-induced podocyte injury. A CCK-8 assay was used in vitro to detect the activity of podocytes subjected to different glucose concentrations. Previous studies reported that podocytes exhibited lower activity under HG stimulation for $48 \mathrm{~h}$ (16). The present study found that, when podocytes were treated with glucose at a concentration of $>40 \mathrm{mM}$ for $48 \mathrm{~h}$, cell viability was decreased significantly (Fig. 4A). LC3 is a soluble protein, which can be hydrolyzed to LC3I to form LC3II, thus reflecting the change in autophagy flow (17). Immunoblotting was used to observe the protein expression of podocin and LC3II/LC3I at different time points in order to determine the association between podocyte injury and autophagy. As shown in Fig. 4B, under HG conditions, podocin expression was decreased in a time-dependent manner, while LC3II/LC3I expression was decreased gradually after $12 \mathrm{~h}$, which indicated that podocyte injury may be associated with a reduction of autophagy.

To observe whether podocyte injury was associated with polyamine metabolism, the present study detected changes in polyamine metabolizing enzymes. As shown in Fig. 4C, immunoblotting revealed decreased ODC expression and increased SSAT expression in HG-induced podocytes. Podocytes were treated with different concentrations of spermine $(1,5,10,20$ and $50 \mu \mathrm{M})$ in HG medium. The results indicated that 5 and $10 \mu \mathrm{M}$ spermine treatment of HG-induced podocytes increased cell viability. However, when the concentration of spermine was $>10 \mu \mathrm{M}$, cell activity was decreased (Fig. 4D). The aforementioned results validated the conditions used in subsequent experiments.

Spermine reduces $H G$-induced apoptosis by activating autophagy in podocytes. To determine the role of autophagy during spermine treatment of podocytes, the effect of rapamycin, which is known to induce autophagy, on podocyte injury was examined. The podocytes were pretreated with rapamycin for $2 \mathrm{~h}$ before HG treatment. In accordance with the results of spermine pretreatment, rapamycin also partially reversed the effects of $\mathrm{HG}$ on cell viability (Fig. 5A), indicating that spermine can also act as an autophagic agonist.

Cell apoptosis was quantified by Hoechst 33342 staining and immunoblotting. It was observed that HG-induced podocytes showed markedly increased nuclear aggregation, DNA fragmentation and caspase-3 cleavage activity (Fig. 5B and E), indicating that HG treatment induced podocytes apoptosis. Next, podocyte injury was evaluated by immunoblotting and immunofluorescence with podocyte-specific markers. There was a significant reduction in nephrin, podocin and $\mathrm{CD}-2 \mathrm{AP}$ expression in the $\mathrm{HG}$ group (Fig. 5C). In agreement with the results of immunoblotting, 

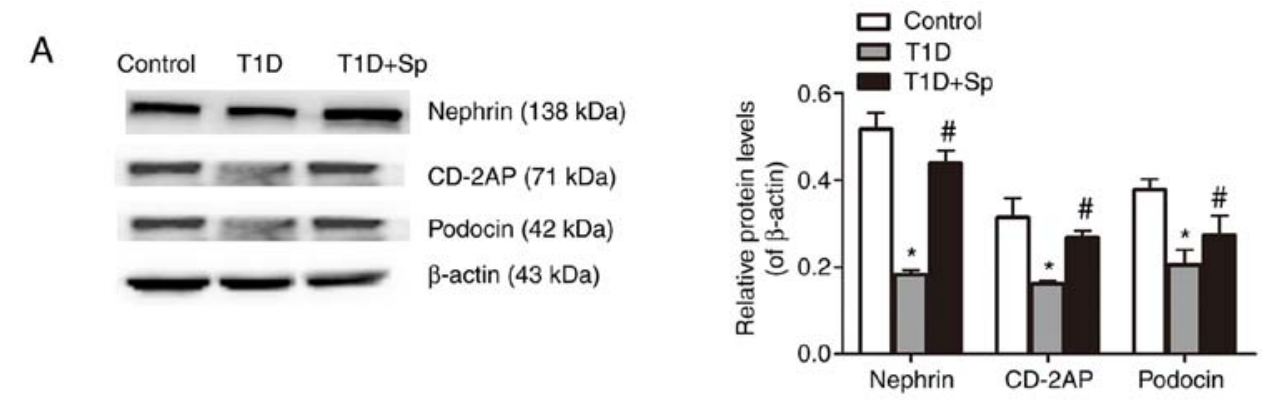
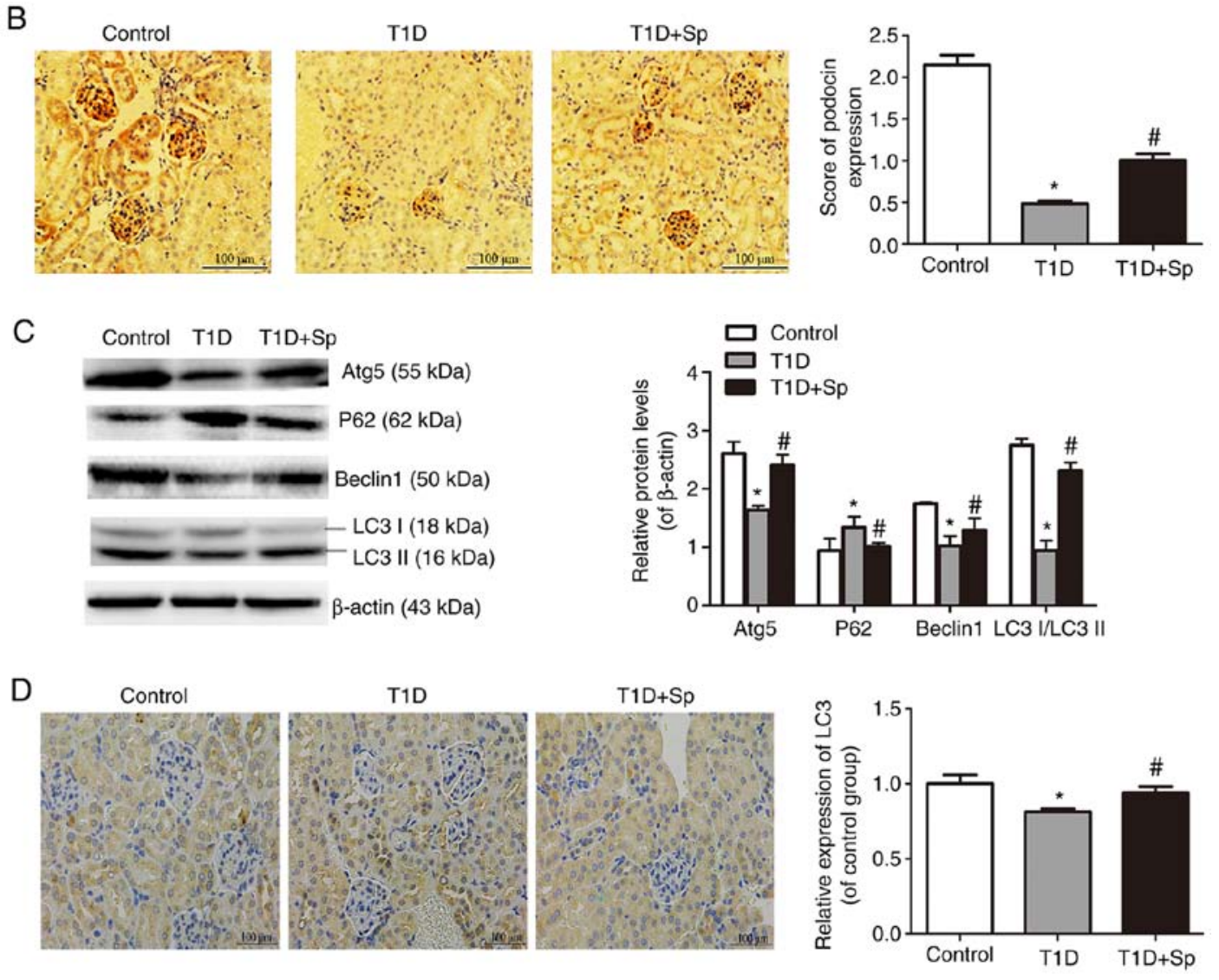

Figure 3. Spermine attenuates podocyte injury and promotes autophagy in T1D rats. (A) Representative immunoblotting of nephrin, CD2-AP and podocin protein levels in kidney sections. (B) Representative immunohistochemical staining for podocin in kidney sections (scale bar, $100 \mu \mathrm{m}$ ) and its quantification. (C) Representative immunoblotting for Atg5, P62, Beclin1 and LC3II/LCI in kidney sections. (D) Representative immunohistochemical staining for LC3 in kidney sections (scale bar, $100 \mu \mathrm{m})$ and its quantification. Data are expressed as the mean \pm standard error of the mean $(\mathrm{n}=6)$. ${ }^{*} \mathrm{P}<0.05$ vs. the control group; ${ }^{\text {"}} \mathrm{P}<0.05$ vs. the T1D group. T1D, type 1 diabetic; CD-2AP, CD2-associated protein; Atg5, autophagy protein 5; LC3, microtube-associated proteins 1A/1B light chain 3; Sp, spermine-treated group.

the data were confirmed by measuring the nephrin-positive area by immunofluorescence staining (Fig. 5D). Finally, to further observe the autophagy of podocytes in vitro, autophagy-related proteins were detected. Immunoblotting showed that Beclin 1 and LC3II/LC3I were decreased, while P62 was increased (Fig. 5E), which was consistent with the in vivo results. Taken together, the aforementioned findings indicated that DN changes were improved by spermine and rapamycin treatment, which suggested that the protective effect of spermine on podocyte injury may be due to a reduction in autophagy.

Spermine protects against $H G$-induced podocyte injury by inhibiting AMPK/mTOR signaling. mTOR kinase is a key molecule in autophagy induction that can be inhibited by activated AMPK. In the present study, p-AMPK and p-mTOR expression were determined by immunoblotting. The results revealed that p-AMPK was decreased and p-mTOR was increased in the HG group. However, spermine and rapamycin reversed the inhibitory effect of HG on AMPK and mTOR activity. The addition of Compound C, an AMPK inhibitor, further confirmed the autophagy-promoting effect of spermine by increasing AMPK phosphorylation and decreasing mTOR phosphorylation (Fig. 6).

\section{Discussion}

The present study mainly demonstrated that hyperglycemia may cause abnormal polyamine metabolism in rat kidney tissue, and further induce podocyte apoptosis and reduction of autophagy, which may be one of the important mechanisms of DN. Exogenous spermine pretreatment was shown to exert 
A

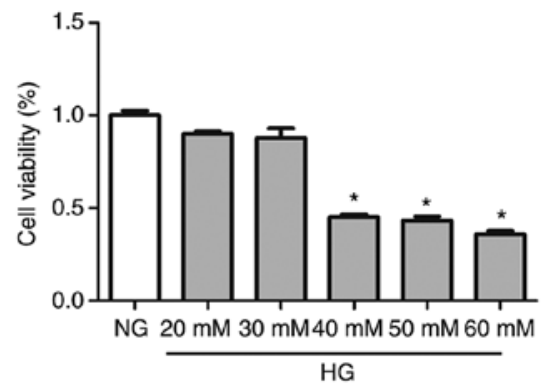

C
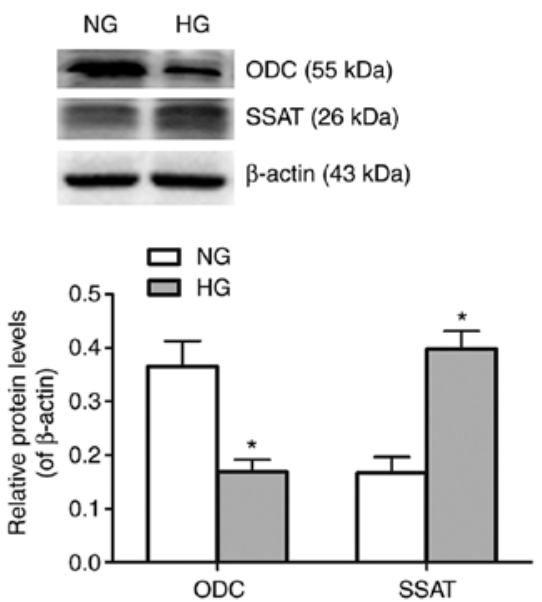

B
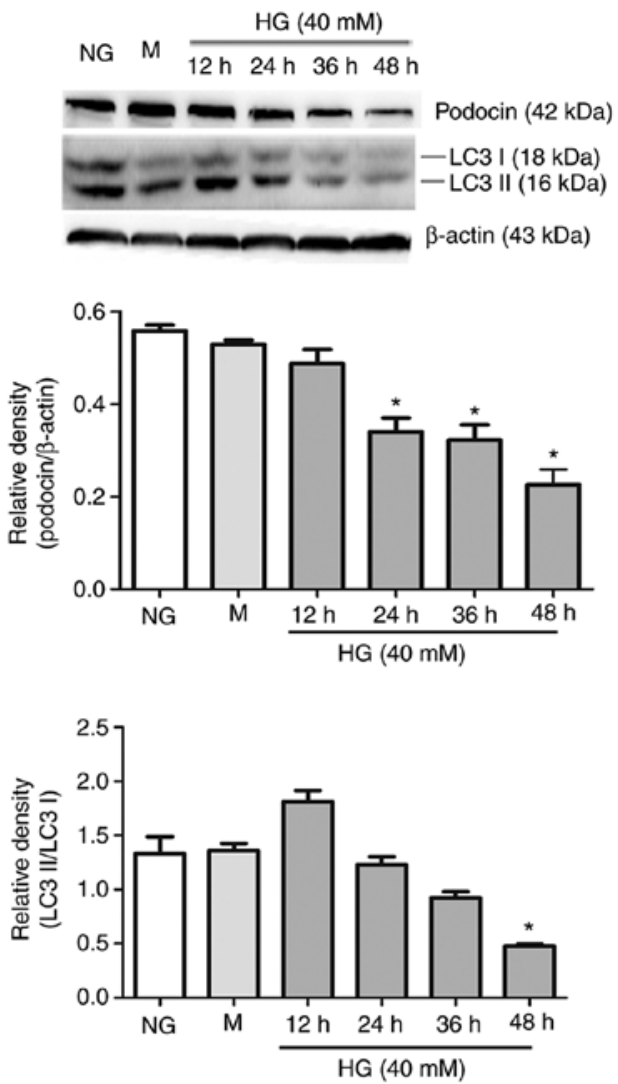

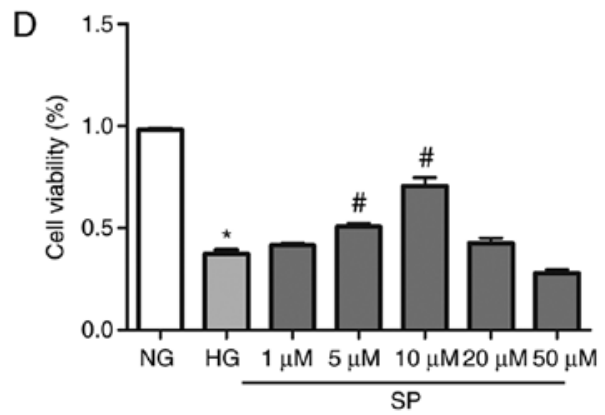

Figure 4. Spermine mitigates HG-induced podocyte injury. (A) CCK-8 assays were performed using podocytes incubated with the indicated concentrations of glucose. (B) Representative immunoblotting analyses of podocin and LC3 protein levels at different times with HG treatment. M was used as an osmotic pressure control. (C) Representative immunoblotting analyses of the protein levels of ODC and SSAT. (D) CCK-8 assays revealed the effect of different concentration of spermine on podocytes under HG conditions. Data are expressed as the mean \pm standard error of the mean $(n=8){ }^{*} \mathrm{P}<0.05$ vs. the NG group; "P<0.05 vs. the HG group. HG, high glucose; CCK-8, Cell Counting Kit-8; M, Mannitol; ODC, ornithine decarboxylase; SSAT, spermidine/spermine N1-acetyltransferase; NG, normal glucose; LC3, microtube-associated proteins 1A/1B light chain 3.

its renoprotective effect by regulating the AMPK/mTOR signaling pathway.

Intraperitoneal injection of STZ was used to establish a diabetic rat model. The blood glucose increased and body weight of the model rats decreased compared with those of the normal control group, indicating that the T1D rat model was successfully established. To explore whether diabetes affected the kidneys, renal function tests and morphological analyses were carried out. The results demonstrated that the Scr and urea content, kidney weight/body weight ratio and UAE were increased. Creatinine and urea are both non-protein nitrogen molecules, and an increase in their levels indicates glomerular filtration dysfunction (18). Under normal conditions, the filtration membrane, which is composed of glomerular capillary endothelial cells, the basement membrane and podocytes, prevents proteins with negative charge and macromolecules from passing through the filtration membrane via mechanical and chemical barriers (19). Increased microalbuminuria indicated that glomerular filtration function was damaged in T1D rats. H\&E and PAS staining further confirmed renal injury (mesangial hyperplasia and matrix accumulation) in the T1D group. Therefore, DN was successfully established in T1D rats, thus providing an experimental platform for further experiments.

Abnormal polyamine metabolism affects gene transcription, translation, expression regulation, autophagy and stress resistance in numerous cellular processes, which has been associated with the occurrence and development of asthma, Parkinson's disease, acute liver injury, acute kidney injury and cancer (20-23). There are two key enzymes in polyamine metabolism: ODC, which is the key enzyme of polyamine synthesis, and SSAT, which is the key enzyme of polyamine degradation $(9,20,23)$. The present results revealed that 

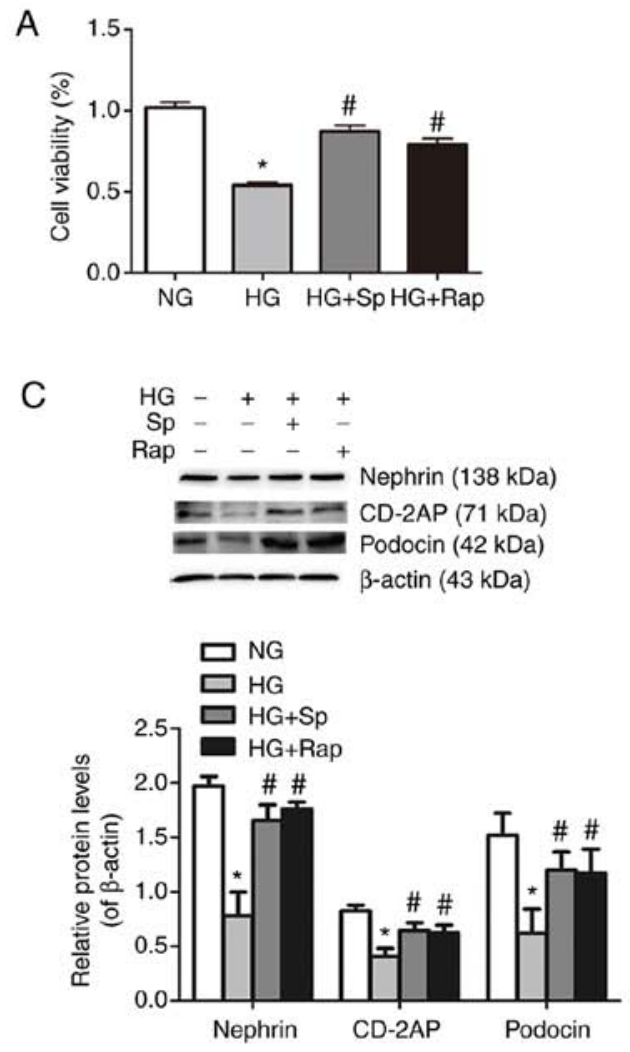

$\mathrm{E}$

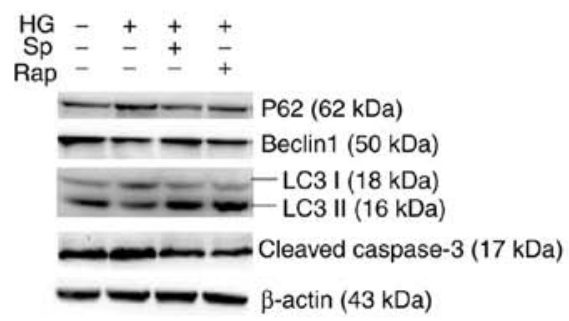

B
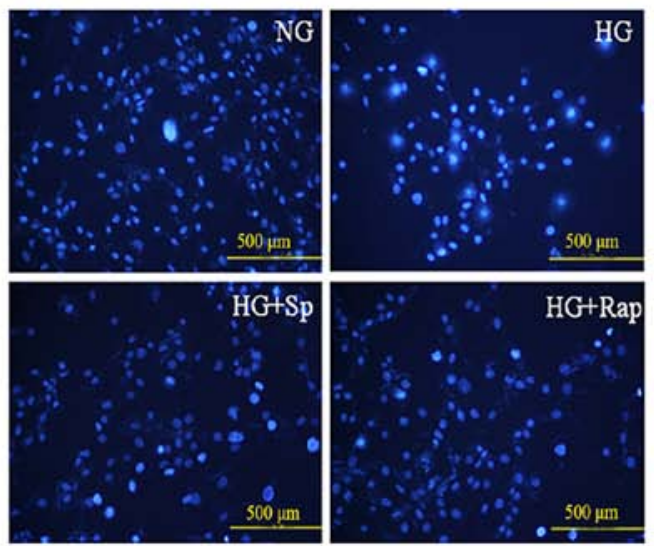

D
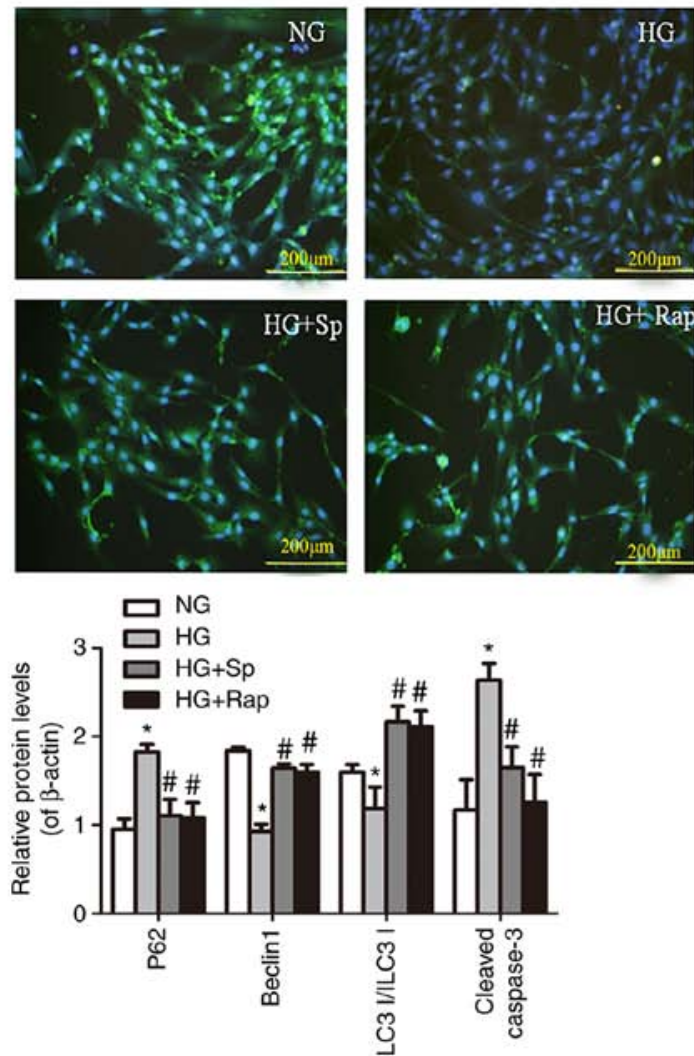

Figure 5. Spermine reduces HG-induced apoptosis via activating autophagy in podocytes. (A) Cell Counting Kit-8 assays were performed using podocytes incubated under HG conditions in the presence of spermine or rapamycin. (B) Hoechst 33342 staining (scale bar, $500 \mu \mathrm{m}$ ). (C) Representative immunoblotting analyses of the proteins levels of nephrin, CD-2AP and podocin protein levels. (D) Representative images of immunofluorescence staining for nephrin under different culture conditions (scale bar, $200 \mu \mathrm{m}$ ). (E) Representative immunoblotting analyses for P62, Beclin1, LC3II/LC3I and cleaved caspase-3. Data are expressed as the mean \pm standard error of the mean $(\mathrm{n}=6-8)$. ${ }^{*} \mathrm{P}<0.05$ vs. the $\mathrm{NG}$ group; ${ }^{\prime \prime} \mathrm{P}<0.05$ vs. the HG group. HG, high glucose; $\mathrm{CD}-2 \mathrm{AP}, \mathrm{CD} 2$-associated protein; LC3, microtube-associated proteins 1A/1B light chain 3; Sp, spermine-treated group; NG, normal glucose.

the expression of ODC decreased, while the expression of SSAT increased, in the renal tissue of DN rats, suggesting that disruption of polyamine metabolism may be implicated in the pathogenesis of DN. Spermine is a multivalent cation of four amino acids with strong activity $(21,25)$. Our previous study demonstrated that spermine preconditioning may improve myocardial infarction and myocardial I/R injury, and plays an important protective role in numerous cardiovascular diseases (26). Therefore, the present study also tried to improve DN by applying exogenous spermine pretreatment.

Since the aforementioned results suggested that hyperglycemia reduces endogenous spermine in T1D rat kidney tissue, exogenous spermine treatment was applied in subsequent experiments. The present results demonstrated that spermine significantly inhibited renal dysfunction, improved morphological damage, reduced the thickness of the GBM and the fusion of the FPs. These results suggested a preliminary protective effect of spermine against DN.

Podocytes are the main components of the glomerular barrier, and FPs are normally arranged on the outside of the GBM and connected laterally via the glomerular slit diaphragm (27). The slit diaphragm constitutes a signaling platform that contains a protein complex of nephrin and podocin, which plays a major role in maintaining the structural and functional integrity of the glomerular filtration barrier (28). 

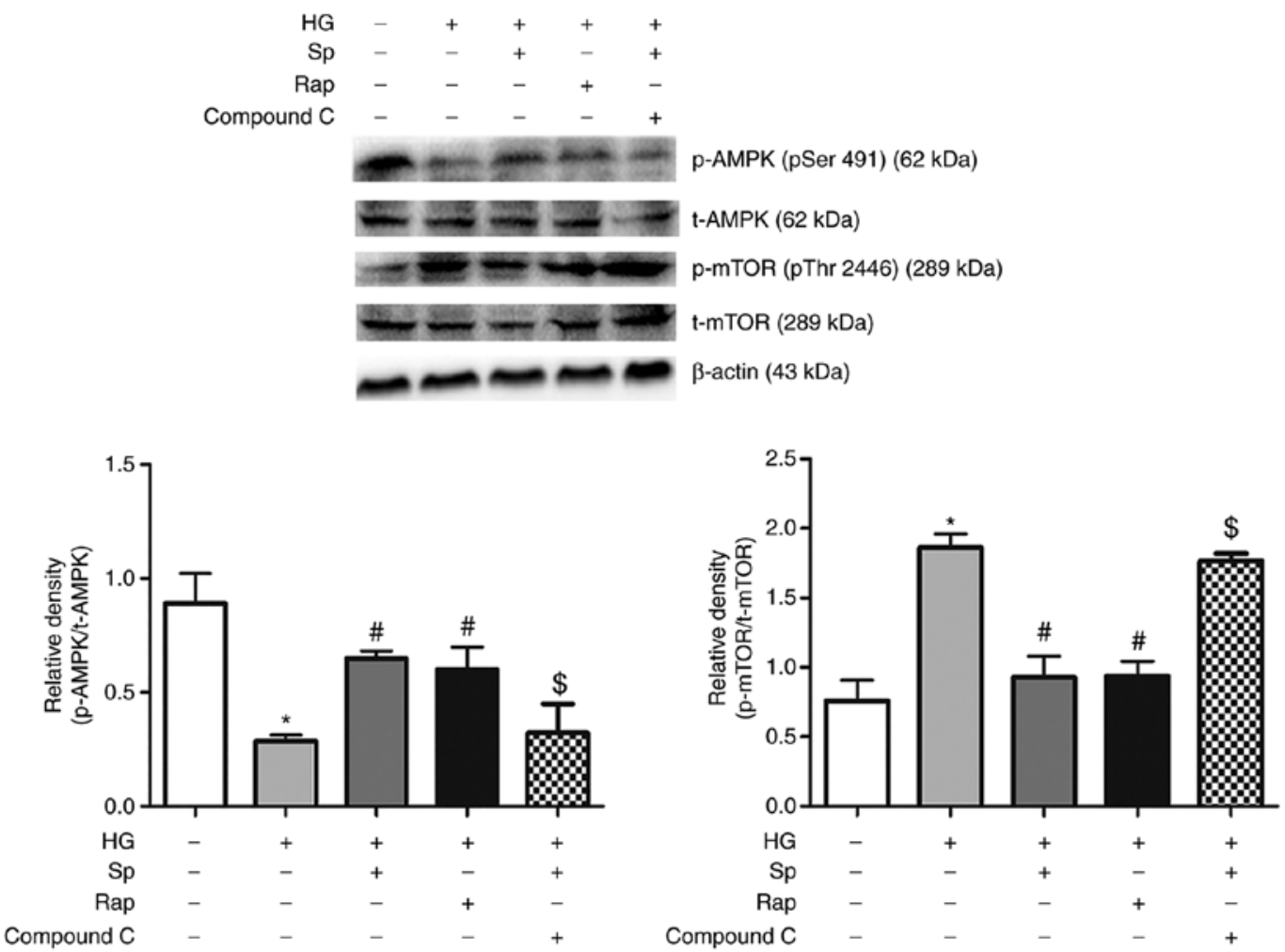

Figure 6. Spermine protects against HG-induced podocyte injury by inhibiting the AMPK/mTOR pathway. Representative immunoblotting analyses of the protein levels of p-AMPK, t-AMPK, p-mTOR and t-mTOR. Data are expressed as the mean \pm standard error of the mean ( $\mathrm{n}=6)$. " $\mathrm{P}<0.05 \mathrm{vs.}$. the NG group; ${ }^{\#} \mathrm{P}<0.05$ vs. the HG group; ${ }^{\mathrm{S}} \mathrm{P}<0.05$ vs. the HG + Sp group. HG, high glucose; $\mathrm{p}-$, phosphorylated; t-, total; Sp, spermine-treated group; NG, normal glucose.

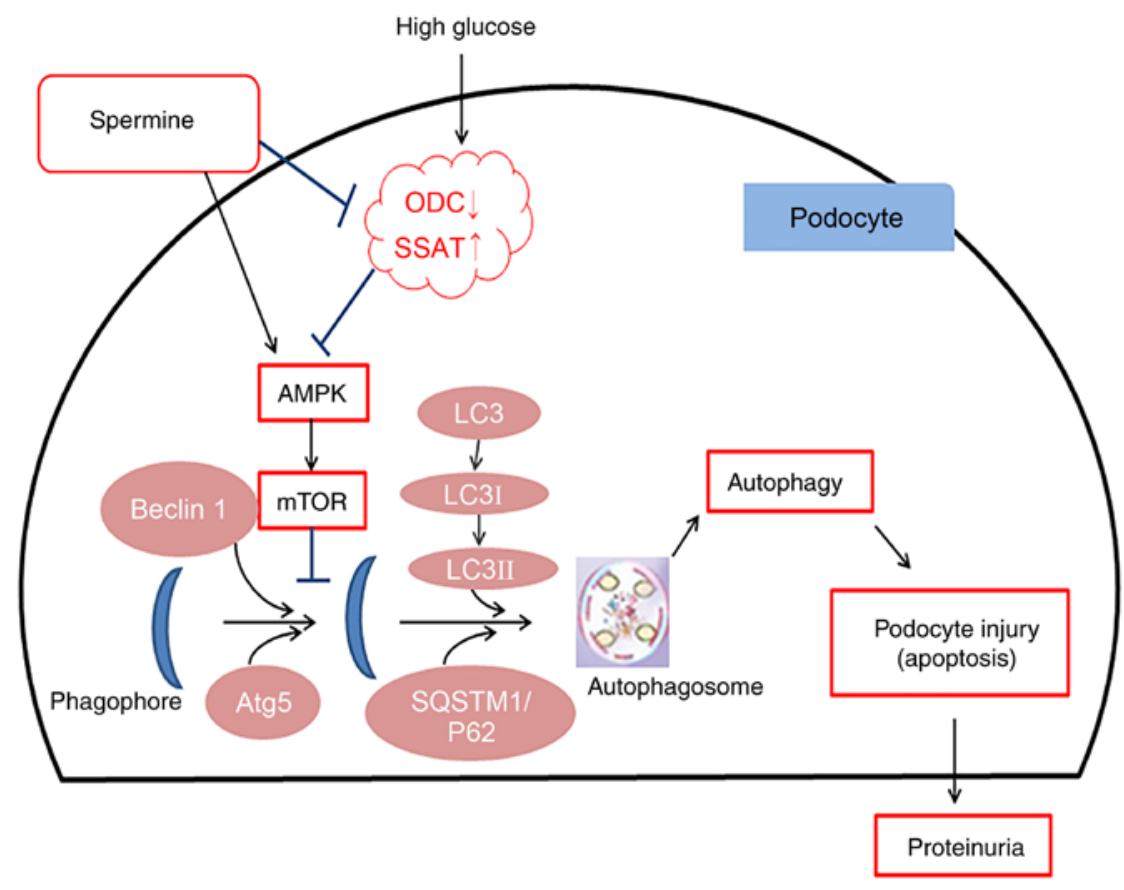

Figure 7. Schematic diagram of the mechanism of the protective effect of spermine against high glucose-induced diabetic podocyte injury. ODC, ornithine decarboxylase; SSAT, spermidine/spermine N1-acetyltransferase; Atg5, autophagy protein 5; LC3, microtube-associated proteins 1A/1B light chain 3.

The slit diaphragm is linked to the actin-based cytoskeleton located in particular parts of podocytes through CD-2AP in FPs $(29,30)$. Therefore, podocyte damage is the main cause of proteinuria. The present study found that nephrin, CD-2AP and podocin levels were markedly reduced in diabetic kidney and HG-treated podocytes, while spermine pretreatment strongly mitigated this reduction. These results suggested that podocyte injury caused by abnormal polyamine metabolism plays a key role in DN, but its underlying mechanism must be further investigated. 
Autophagy is involved in the regulation of cell differentiation, development, nutritional metabolism and maintenance of the cell environment by transferring damaged organelles and misfolded proteins to lysosomes for degradation $(31,32)$. The damage caused by autophagy leads to the loss of podocytes and extensive proteinuria in DN (33). In the present study, changes in the corresponding autophagy indices were observed in diabetic kidney and HG-induced podocytes. The results demonstrated that spermine pretreatment could increase autophagy activation by increasing the levels of Atg5, LC3-II/LC3-I ratio and Beclin 1, and by reducing the accumulation of P62, which indicated that reduced autophagy caused by abnormal polyamine metabolism is an intrinsic cause of diabetic podocyte dysfunction. Exogenous spermine may play a protective role by restoring autophagy.

Autophagy in podocytes is a complex process that can be controlled by several signaling pathways, with AMPK/mTOR signaling being one of the major pathways that regulates autophagy. mTOR and AMPK belong to the serine/threonine protein kinase family, which plays a major role in maintaining blood glucose, body energy levels and homeostasis (34). mTOR is an upstream regulatory protein of the autophagy pathway, and increased activity of mTOR can inhibit downstream autophagy signaling proteins $(35,36)$. Excessive mTOR activity in podocytes leads to dysregulation of autophagy, resulting in autophagic cell death, which plays a major role in the development of DN $(37,38)$. Podocyte maintenance is safeguarded by the mTOR pathway, which is known to inhibit autophagy (39). Early findings in the glomeruli included depletion of energy equivalents, which resulted in increased AMPK signaling and changes in the proteins that regulate the metabolites (40). The present experiments also revealed that, in HG-induced podocytes, p-AMPK decreased and p-mTOR increased, which suggested the inhibition of autophagy. Spermine and rapamycin (an autophagy agonist) alleviated the inhibition of autophagy, whereas these effects were reversed by the effect of Compound C (an AMPK inhibitor). That is to say, spermine can protect against HG-induced podocyte injury by activating autophagy through regulation of the AMPK/mTOR signaling pathway.

In conclusion, in the present study, abnormal polyamine metabolism disorders were observed in both T1D rats and HG-treated podocytes, which in turn triggered apoptosis and reduced autophagy, resulting in podocyte injury and extensive albuminuria. Exogenous spermine played a protective role by regulating AMPK/mTOR signaling to restore autophagy (Fig. 7). These findings may provide theoretical and experimental grounds for the prevention and treatment of DN, and support the clinical application of spermine.

\section{Acknowledgements}

Not applicable.

\section{Funding}

The present research was supported by the Scientific Research Fund of Harbin Medical University-Daqing (grant no. DQXN201607), the Joint guiding project of Natural Science Foundation of Heilongjiang Province (grant no. LH2019H045), the National Natural Science Foundation of China (grant no. 81800260) and University Nursing Program for Young Scholars with Creative Talents in Heilongjiang Province (grant no. UNPYSCT-2020165).

\section{Availability of data and materials}

The datasets used and/or analyzed during the current study are available from the corresponding author on reasonable request.

\section{Authors' contributions}

$\mathrm{CW}, \mathrm{CX}, \mathrm{XZ}$ and $\mathrm{LZ}$ designed the research and drafted the manuscript. ZC, SL, BC, NW and JC completed the experiments and data analysis. $\mathrm{CX}$ and $\mathrm{CW}$ confirmed the authenticity of all the raw data. All authors read and approved the final manuscript.

\section{Ethics approval and consent to participate}

All animal experimental protocols complied with the Guide for the Care and Use of Laboratory Animals published by the National Institutes of Health. The present study was approved by the Institutional Animal Research Committee of Harbin Medical University (Harbin, China).

\section{Patient consent for publication}

Not applicable.

\section{Competing interests}

The authors declare that they have no competing interests.

\section{References}

1. Saran R, Li Y, Robinson B, Ayanian J, Balkrishnan R, Bragg-Gresham J, Chen JT, Cope E, Gipson D, He K, et al: US renal data system 2014 annual data report: Epidemiology of kidney disease in the united states. Am J Kidney Dis 66 (Suppl 1): Svii, S1-305, 2015.

2. Xiao L, Wang M, Yang S, Liu F and Sun L: A glimpse of the pathogenetic mechanisms of Wnt/ $\beta$-catenin signaling in diabetic nephropathy. Biomed Res Int 2013: 987064, 2013.

3. Brownlee M: The pathobiology of diabetic complications: A unifying mechanism. Diabetes 54: 1615-1625, 2005.

4. Giacco F and Brownlee M: Oxidative stress and diabetic complications. Circ Res 107: 1058-1070, 2010.

5. Bae DH, Lane DJR, Jansson PJ and Richardson DR: The old and new biochemistry of polyamines. Biochim Biophys Acta Gen Subj 1862: 2053-2068, 2018.

6. Wallace HM, Fraser AV and Hughes ZA: A perspective of polyamine metabolism. Biochem J 376: 1-14, 2003.

7. Michael AJ: Polyamines in eukaryotes, bacteria, and archaea J Biol Chem 291: 14896-14903, 2016.

8. Kalac P: Health effects and occurrence of dietary polyamines: A review for the period 2005-mid 2013. Food Chem 161: 27-39, 2014.

9. Lane DJR, Bae DH, Siafakas AR, Suryo Rahmanto Y, Al-Akra L, Jansson PJ, Casero RA Jr and Richardson DR: Coupling of the polyamine and iron metabolism pathways in the regulation of proliferation: Mechanistic links to alterations in key polyamine biosynthetic and catabolic enzymes. Biochim Biophys Acta Mol Basis Dis 1864: 2793-2813, 2018.

10. Zahedi K, Barone S, Wang Y, Murray-Stewart T, Roy-Chaudhury P, Smith RD, Casero RA Jr and Soleimani M: Proximal tubule epithelial cell specific ablation of the spermidine/spermine N1-acetyltransferase gene reduces the severity of renal ischemia/reperfusion injury. PLoS One 9: e110161, 2014. 
11. Igarashi $\mathrm{K}$ and Kashiwagi K: Modulation of cellular function by polyamines. Int J Biochem Cell Biol 42: 39-51, 2010.

12. Wang J, Li S, Wang J, Wu F, Chen Y, Zhang H, Guo Y, Lin Y, Li L, $\mathrm{Yu} \mathrm{X}$, et al: Spermidine alleviates cardiac aging by improving mitochondrial biogenesis and function. Aging (Albany NY) 12: 650-671, 2020

13. Mason TJ and Matthews M: Aquatic environment, housing, and management in the eighth edition of the Guide for the care and use of laboratory animals: Additional considerations and recommendations. J Am Assoc Lab Anim Sci 51: 329-32, 2012.

14. Wang Y, Chen J, Li S, Zhang X, Guo Z, Hu J, Shao X, Song N, Zhao Y, Li H, et al: Exogenous spermine attenuates rat diabetic cardiomyopathy via suppressing ROS-p53 mediated downregulation of calcium-sensitive receptor. Redox Biol 32: 101514, 2020.

15. Tsai HJ, Liao MH, Shih CC, Ka SM, Tsao CM and Wu CC: Angiotensin-(1-7) attenuates organ injury and mortality in rats with polymicrobial sepsis. Crit Care 22: 269, 2018.

16. Chen Y, Liu Q, Shan Z, Mi W, Zhao Y, Li M, Wang B, Zheng X and Feng W: Catalpol ameliorates podocyte injury by stabilizing cytoskeleton and enhancing autophagy in diabetic nephropathy. Front Pharmacol 10: 1477, 2019.

17. Choi ME: Autophagy in kidney disease. Annu Rev Physiol 82 287-322, 2020.

18. Medler S and Harrington F: Measuring dynamic kidney function in an undergraduate physiology laboratory. Adv Physiol Educ 37: 384-91, 2013.

19. Reiser J and Sever S: Podocyte biology and pathogenesis of kidney disease. Annu Rev Med 64: 357-366, 2013.

20. Wang W, Zhang H, Xue G, Zhang L, Zhang W, Wang L, Lu F, $\mathrm{Li} \mathrm{H}$, Bai S, Lin Y, et al: Exercise training preserves ischemic preconditioning in aged rat hearts by restoring the myocardial polyamine pool. Oxid Med Cell Longev 2014: 457429, 2014.

21. Jain V, Raina S, Gheware AP, Singh R, Rehman R, Negi V, Murray Stewart T, Mabalirajan U and Mishra AK: Reduction in polyamine catabolism leads to spermine-mediated airway epithelial injury and induces asthma features. Allergy 73 : 2033-2045, 2018.

22. Green DR: Polyamines and aging: A CLEAR connection? Mol Cell 76: 5-7, 2019.

23. Zahedi K, Barone S and Soleimani M: Polyamine catabolism in acute kidney injury. Int J Mol Sci 20: 4790, 2019.

24. Mollet G, Ratelade J, Boyer O, Muda AO, Morisset L, Lavin TA, Kitzis D, Dallman MJ, Bugeon L, Hubner N, et al: Podocin inactivation in mature kidneys causes focal segmental glomerulosclerosis and nephrotic syndrome. J Am Soc Nephrol 20: 2181-2189, 2009.

25. Russell DH: Clinical relevance of polyamines. Crit Rev Clin Lab Sci 18: 261-311, 1983.

26. Wei C, Li H, Wang Y, Peng X, Shao H, Li H, Bai S and Xu C: Exogenous spermine inhibits hypoxia/ischemia-induced myocardial apoptosis via regulation of mitochondrial permeability transition pore and associated pathways. Exp Biol Med (Maywood) 241: 1505-1515, 2016
27. Ying $\mathrm{Q}$ and $\mathrm{Wu} \mathrm{G}$ : Molecular mechanisms involved in podocyte EMT and concomitant diabetic kidney diseases: An update. Ren Fail 39: 474-483, 2017.

28. Asanuma $\mathrm{K}$ and Mundel P: The role of podocytes in glomerular pathobiology. Clin Exp Nephrol 7: 255-259, 2003.

29. Tolvanen TA, Dash SN, Polianskyte-Prause Z, Dumont V and Lehtonen S: Lack of CD2AP disrupts Glut4 trafficking and attenuates glucose uptake in podocytes. J Cell Sci 128: 4588-4600, 2015.

30. Ristola $\mathrm{M}$ and Lehtonen S: Functions of the podocyte proteins nephrin and Neph3 and the transcriptional regulation of their genes. Clin Sci (Lond) 126: 315-328, 2014.

31. Turkmen K: Inflammation, oxidative stress, apoptosis, and autophagy in diabetes mellitus and diabetic kidney disease: The four horsemen of the apocalypse. Int Urol Nephrol 49: 837-844, 2017.

32. Yang D, Livingston MJ, Liu Z, Dong G, Zhang M, Chen JK and Dong Z: Autophagy in diabetic kidney disease: Regulation, pathological role and therapeutic potential. Cell Mol Life Sci 75: 669-688, 2017.

33. Yasuda-Yamahara M, Kume S, Tagawa A, Maegawa $H$ and Uzu T: Emerging role of podocyte autophagy in the progression of diabetic nephropathy. Autophagy 11: 2385-2386, 2015.

34. Szrejder M and Piwkowska A: AMPK signalling: Implications for podocyte biology in diabetic nephropathy. Biol Cell 111: 109-120, 2019.

35. Li W, Zhu L, Ruan ZB, Wang MX, Ren Y and Lu W: Nicotinamide protects chronic hypoxic myocardial cells through regulating mTOR pathway and inducing autophagy. Eur Rev Med Pharmacol Sci 23: 5503-5511, 2019.

36. Miao H, Qiu F, Huang B, Liu X, Zhang H, Liu Z, Yuan Y, Zhao Q, Zhang H, Dong $\mathrm{H}$ and Zhang Z: PKC $\alpha$ replaces AMPK to regulate mitophagy: Another PEDF role on ischaemic cardioprotection. J Cell Mol Med 22: 5732-5742, 2018.

37. Li Z, Li Q, Lv W, Jiang L, Geng C, Yao X, Shi X, Liu Y and Cao J: The interaction of Atg4B and Bcl-2 plays an important role in $\mathrm{Cd}$-induced crosstalk between apoptosis and autophagy through disassociation of Bcl-2-Beclin1 in A549 cells. Free Radic Biol Med 130: 576-591, 2019.

38. Levine B and Kroemer G: Autophagy in the pathogenesis of disease. Cell 132: 27-42, 2008.

39. Bork T, Liang W, Yamahara K, Lee P, Tian Z, Liu S, Schell C, Thedieck K, Hartleben B, Patel K, et al: Podocytes maintain high basal levels of autophagy independent of mtor signaling. Autophagy 16: 1932-1948, 2020.

40. Rinschen MM, Palygin O, Guijas C, Palermo A, Palacio-Escat N Domingo-Almenara X, Montenegro-Burke R, Saez-Rodriguez J, Staruschenko A and Siuzdak G: Metabolic rewiring of the hypertensive kidney. Sci Signal 12: eaax9760, 2019.

This work is licensed under a Creative Commons Attribution-NonCommercial-NoDerivatives 4.0 International (CC BY-NC-ND 4.0) License. 